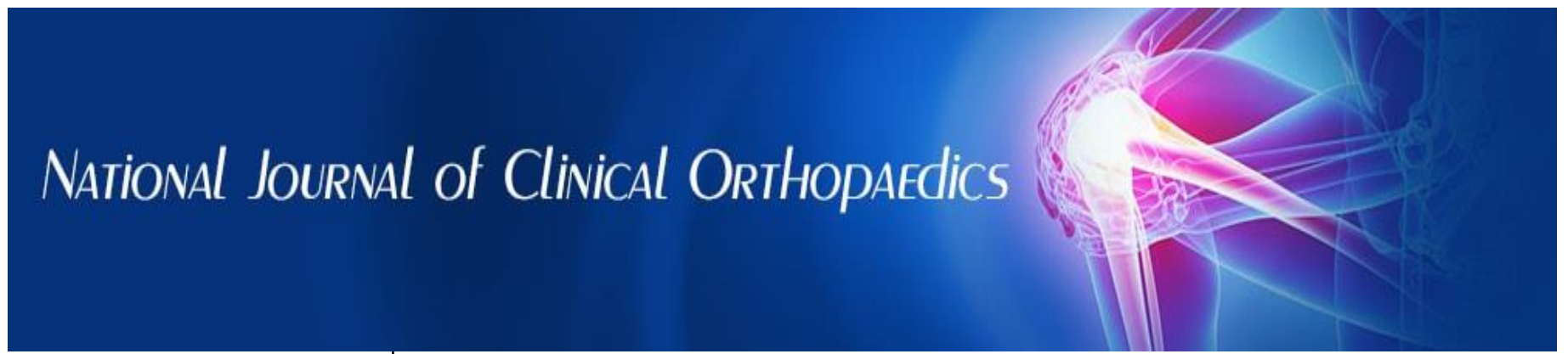

ISSN (P): 2521-3466

ISSN (E): 2521-3474

(C) Clinical Orthopaedics www.orthoresearchjournal.com 2021; 5(1): 106-108

Received: 14-11-2020

Accepted: 29-12-2020

Dr. Shreesh Kadur JM MBBS, MS DNB, Department of Orthopaedic Surgeon, Sky Superspeciality Hospital, Iritty, Kannur, Kerala, India

Dr. Sharvani S

MBBS, MD, Senior Resident, Kodagu Institute of Medical Sciences, Madikeri, Karnataka, India
Corresponding Author: Dr. Shreesh Kadur JM MBBS, MS DNB, Department of Orthopaedic Surgeon, Sky Superspeciality Hospital, Iritty, Kannur, Kerala, India

\section{Multiarticular chronic Tophaceous Gout: A benign disorder with malignant presentation}

\section{Dr. Shreesh Kadur JM and Dr. Sharvani S}

DOI: https://doi.org/10.33545/orthor.2021.v5.i1b.270

\begin{abstract}
Gout is a metabolic disorder caused by deposition of MSU (monosodium urate crystals) in joints and associated inflammatory reaction to it. It usually affects elderly men and postmenopausal women. Chronic tophaceous gout takes usually 10 years to appear due to recurrent episodes of gouty arthritis. Our case is a 55 year male person with multiarticular tophaceous gouty arthritis. Pharmacological treatment has high remittance rate. Surgical treatment is seldom required for gout and is usually reserved for cases of recurrent attacks with deformities, severe pain, infection and joint destruction. Our case is interesting due to the fact that it is early onset and treated only by pharmacological means.
\end{abstract}

Keywords: Gout, uric acid, Tophaceous

\section{Introduction}

Gout is a common pathology due to defective uric acid metabolism charcterised by spectrum of presentations such as acute gout, recurrent episodes of inflammatory arthritis, tophaceous soft tissue deposits of monosodium urate crystals, uric acid renal calculi and chronic nephropathy. Manifestations commonly appear as firm, pink nodules or fusiform swellings ${ }^{[1}$ ${ }^{2]}$. We present a case of multiarticular chronic tophaceous gout in 55 year old adult male.

\section{Case report}

Our patient, 55 year male presented to us with history of multiple swelling over the both the hands and the feet There was history of discharge of chalky white material from some the swellings, especially hands. (Figure 1,2) The swellings developed gradually over 4 year duration. Patient had no family history but, there was history of high protein diet and alcohol from past 20 years. He had tried multiple traditional methods for the condition but never resolved. He had difficulty to walk and even perform routine daily activities. Radiographs of both hands showed soft-tissue swelling and periarticular erosions in metacarpophalyngeal joints with destruction of the same.(figure 3) Radiographs of the foot showed soft tissue swelling and partial erosion of the first MTP joint.(figure 4) Blood investigations showed raised serum uric acid levels $(10 \mathrm{mg} / \mathrm{dl})$ and ESR $(55 \mathrm{~mm} / \mathrm{hr})$. FNAC of the swellings showed predominantly needle like crystals in acellular background.( figure 5) Patient was put on colchicine $1 \mathrm{mg}$ / day in divided doses, short term steroids for few days and NSAIDS for a week. It was followed by febuxostat $40 \mathrm{mg}$ / day for next four weeks. In a week time pain reduced drastically and swelling reduced to 50 percent of its size in 3 weeks time. Patient was able to do his routine daily activities. After 4 weeks uric acid levels came upto $6.7 \mathrm{mg} / \mathrm{dl}$. Subsequently patient was lost for follow up. 


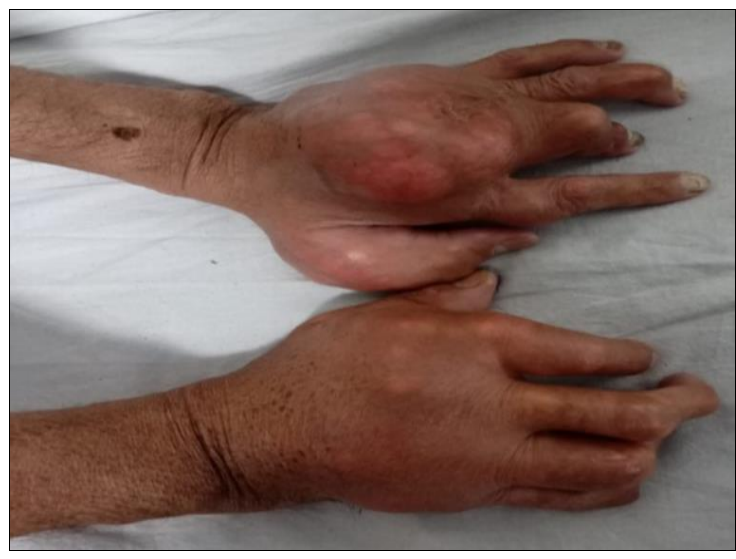

Fig 1: Clinical picture of both hand showing tophaceous firm swelling

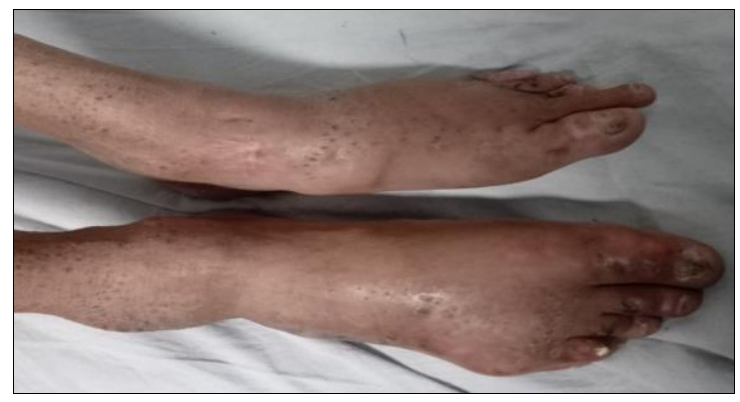

Fig 2: Clinical picture of both feet showing swelling

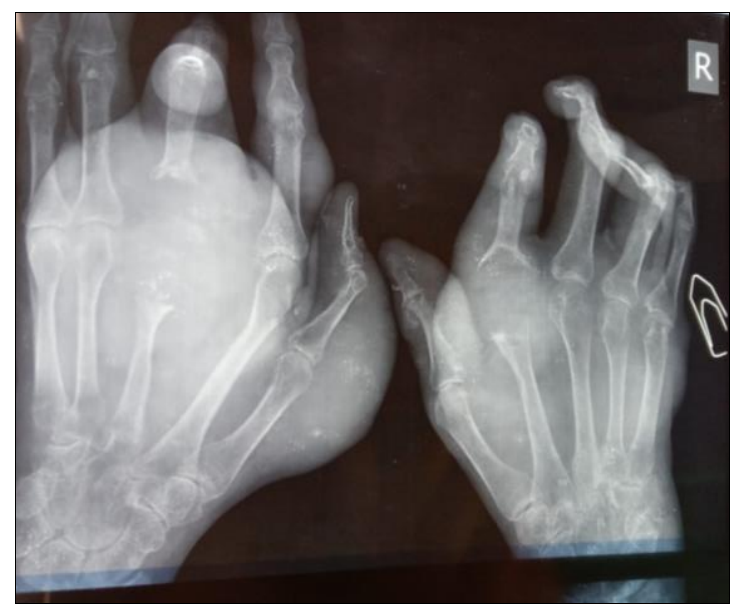

Fig 3: Xray picture of hand showing periarticular destruction of MCP joint

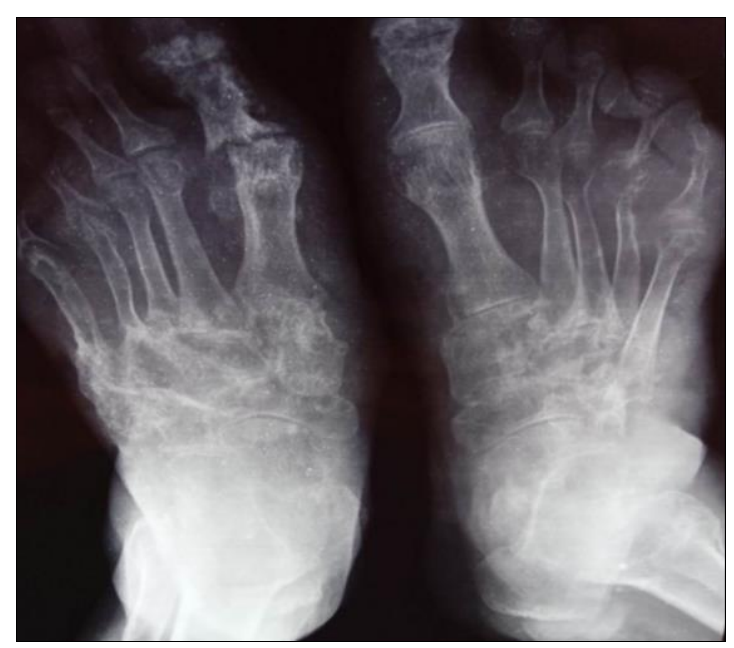

Fig 4: Xray picture of feet showing periarticular erosion in $1^{\text {st }} \mathrm{mtp}$ jopint

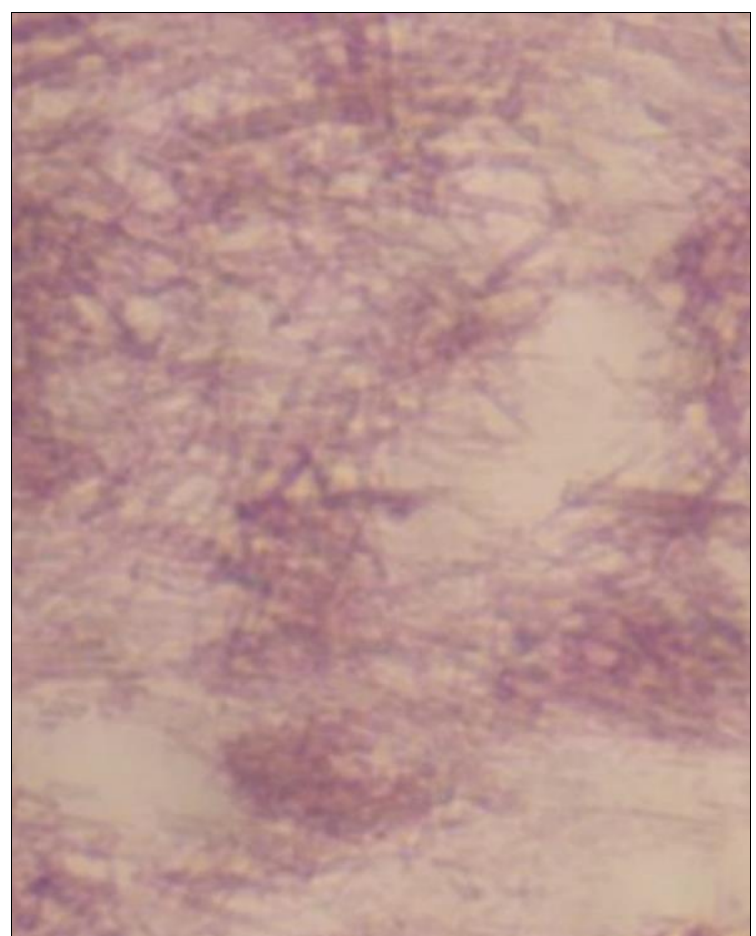

Fig 5: Light microscopy picture showing uric acid presnting as needle like crystals

\section{Discussion}

Gout is a common disease caused due to abnormal uric acid metabolism. The uric acid gets deposited in the joints due to crystallization resulting in recurrent arthritis. Older age, male sex, postmenopausal women, and black race are at higher risk of developing gout ${ }^{[3]}$.

Hyperuricemia and gout can be attributed to uric acid elevating drugs (diuretics, cyclosporine, low doses of aspirin, etc.), genetic Ppolymorphisms in genes controlling renal urate transport and predisposing dietary factors, such as consumption of red meat, seafood, and alcohol ${ }^{[4]}$.

Gout usually starts with affecting the first metatarsophalangeal joint of the foot and less commonly other joints. In untreated and traditionally treated patients tophaceous gout may develop, which is characterized by chronic destructive polyarticular involvement and tophi. Chronic tophaceous gout frequently occurs after 10 years or more of recurrent polyarticular gout ${ }^{[5]}$. Tophi can occur in soft tissue and different organs either in presence or in absence of gouty arthritis. The prevalence of gout is much higher in men than in women and rises with age due to uricosuric action of estrogen.

In our case, history was quite short with extensive tophi in hands and feet occurring within 4 years of onset of arthritis. Kim et al. reported disseminated miliarial gout in a 34-year-old male, which occurred 4 years after onset of gouty arthritis ${ }^{[6]}$. Jung $e t$ $a l$. reported a case of disseminated gout in the form of nodules that appeared after 10 years of gouty arthritis ${ }^{[7]}$. Gout can affect the large joints which can be confused with rheumatoid arthritis and calcinosis cuts. However it can be differentiated neurologically, biochemically and radiologically.

Treatment of Gout should include dietary modification as well as pharmacological measures. Non pharmacological measures include diet restriction of purines, reducing intake of alcohol, and reducing weight. An acute attack is treated by NSAIDs, colchicines, or steroids. Normalization of hyperuricemia is the aim of treatment in chronic tophaceous gout. Drugs like probenecid favoring excretion of uric acid or allopurinol and 
febuxostat for inhibiting production of uric acid are used. Surgical removal of tophi is attempted only in the case of severe pain, joint deformities, or physical removal of tophi ${ }^{[8]}$.

Statement of consent: Informed written consent was taken from the patient for the publication of educational purpose.

\section{Conflict of interest: None}

\section{References}

1. Fam AG, Assaad D. Intradermal urate tophi. The Journal of rheumatology 1997;24(6):1126-31.

2. Li EK. Gout: a review of its aetiology and treatment. Hong Kong medical journal=Xianggang Yi Xue Za Zhi 2004;10(4):261-70.

3. Shukla R, Vender RB, Alhabeeb A, Salama S, Murphy F. Miliarial gout (a new entity). Journal of cutaneous medicine and surgery 2007;11(1):31-4.

4. Lee SJ, Terkeltaub RA, Kavanaugh A. Recent developments in diet and gout. Current opinion in rheumatology 2006;18(2):193-8.

5. Iacobellis G. A rare and asymptomatic case of mitral valve tophus associated with severe gouty tophaceous arthritis. Journal of endocrinological investigation 2004;27(10):9656.

6. Kim M, Lee SI, Cheon YH. Unusual milia-type intradermal tophi in a patient with gout. The Korean journal of internal medicine 2020;35(3):736.

7. Jung HY, Yu DS, Kim JW, Jang ED. Disseminated cutaneous gout: A rare clinical presentation. Indian Journal of Dermatology, Venereology, and Leprology 2016;82(2):204.

8. Verma S, Bhargav P, Toprani T, Shah V. Multiarticular tophaceous gout with severe joint destruction: A pictorial overview with a twist. Indian journal of dermatology 2014;59(6):609. 